\title{
Carrier screening for spinal muscular atrophy
}

Thomas W. Prior, PhD, for the Professional Practice and Guidelines Committee

Key Words: spinal muscular atrophy, carrier screening, genetic testing, population screening

Disclaimer: This guideline is designed primarily as an educational resource for health care providers to help them provide quality medical genetic services. Adherence to this guideline does not necessarily assure a successful medical outcome. This guideline should not be considered inclusive of all proper procedures and tests or exclusive of other procedures and tests that are reasonably directed to obtaining the same results. In determining the propriety of any specific procedure or test, the geneticist should apply his or her own professional judgment to the specific clinical circumstances presented by the individual patient or specimen. It may be prudent, however, to document in the patient's record the rationale for any significant deviation from this guideline.

The autosomal recessive disorder proximal spinal muscular atrophy (SMA, MIM \#253300) is a severe neuromuscular disease characterized by degeneration of alpha motor neurons in the spinal cord, which results in progressive proximal muscle weakness and paralysis. SMA is the second most common fatal autosomal recessive disorder after cystic fibrosis, with an estimated prevalence of 1 in 10,000 live births and a carrier frequency of $1 / 40-1 / 60$. Childhood SMA is subdivided into three clinical groups on the basis of age of onset and clinical course: type I SMA (Werdnig-Hoffmann) is characterized by severe, generalized muscle weakness and hypotonia at birth or within the first 3 months. Death from respiratory failure usually occurs within the first 2 years. Children with type II are able to sit, although they cannot stand or walk unaided and survive beyond 4 years. Type III SMA (Kugelberg-Welander) is a milder form, with onset during infancy or youth: patients learn to walk unaided.

The survival motor neuron $(S M N)$ gene comprises nine exons and has been shown to be the primary SMA determining gene. Two almost identical $S M N$ genes are present on $5 \mathrm{q} 13$ : the telomeric or SMN1 gene, which is the SMA-determining gene, and the centromeric or SMN2 gene. The SMN1 gene exon 7 is homozygously absent in approximately $95 \%$ of affected patients, with few exceptions, the remainder are heterozygous for the exon 7 deletion and a small more subtle mutation in the other allele (compound heterozygotes). Although abnormalities of the SMN1 gene are observed in the majority of patients, no phenotype-genotype correlation was observed because

From the Department of Pathology, The Ohio State University, Columbus, Ohio. Thomas W. Prior, PhD, Department of Pathology, The Ohio State University, 125 Hamilton Hall, 1645 Neil Avenue, Columbus, OH 43210. E-mail: Thomas.Prior@osumc.edu.

Disclosure: The author does not have a commercial interest in SMA carrier testing, but does perform the testing in his laboratory.

Submitted for publication August 1, 2008.

Accepted for publication August 1, 2008.

DOI: 10.1097/GIM.0b013e318188d069
SMN1 exon 7 is absent in the majority of patients independent of the type of SMA. This is because routine diagnostic methods do not distinguish between a deletion of SMN1 and a conversion event whereby SMN1 is replaced by a copy of SMN2. There have now been several studies that have shown that the SMN2 copy number influences the severity of the disease. The copy number varies from zero to three copies in the normal population, with approximately $15 \%$ of normal individuals having no SMN2. However, milder patients with type II or III have been shown to have more copies of SMN2 than do type I patients. It has been proposed that the extra $S M N 2$ in the more mildly affected patients arise through gene conversions, whereby the SMN2 gene is copied either partially or totally into the telomeric locus.

Five base pair changes exist between SMN1 and SMN2 transcripts, and none of these differences change amino acids. Because virtually all SMA individuals have at least one SMN2 gene copy, it was initially not understood why individuals with SMN1 mutations have a SMA phenotype. It has now been shown that the SMN1 gene produces predominately fulllength transcript, whereas the SMN2 copy produces predominately an alternatively transcribed (exon 7 deleted) product. The inclusion of exon 7 in SMN1 transcripts and exclusion of this exon in SMN2 transcripts is caused by a single nucleotide difference at +6 in $S M N$ exon 7. Although the $\mathrm{C}$ to $\mathrm{T}$ change in SMN2 exon 7 does not change an amino acid, it does disrupt an exonic splicing enhancer that results in the majority of SMN2 transcripts lacking exon 7. Therefore, SMA arises because the SMN2 gene cannot fully compensate for the lack of SMN1 expression when SMN1 is mutated. However, the small amount of full length transcripts generated by SMN2 is able to produce a milder type II or III phenotype when the copy number of SMN2 is increased.

The molecular diagnosis of the SMA consists of the detection of the absence of exon 7 of the SMN1 gene. The homozygous absence of detectable SMN1 in SMA patients is being used as a powerful diagnostic test for SMA. Although the targeted 
mutation analysis has an excellent sensitivity of approximately 95\% in identifying affected homozygotes, it cannot detect SMA carriers who have heterozygous deletions of SMN1. Rather, $S M N 1$ gene dosage analysis is required to detect carriers and is highly accurate when performed in an experienced laboratory. Because SMA is one of the most common lethal genetic disorders, with a carrier frequency of $1 / 40-1 / 60$, direct carrier dosage testing has been beneficial to many families with affected children. A number of quantitative polymerase chain reaction assays have been used for the identification of SMA carriers.

There are two limitations of the carrier test. First, approximately $2 \%$ of SMA cases arise as the result of de novo mutation events, which is high when compared with most autosomal recessive disorders. The high rate of de novo mutations in SMN1 may account for the high carrier frequency in the general population despite the genetic lethality of the type I disease. The large number of repeated sequences around the $S M N 1$ and SMN2 locus likely predispose this region to unequal crossovers and recombination events and results in the high de novo mutation rate. The de novo mutations have been shown to occur primarily during paternal meiosis. Second, the copy number of SMN1 can vary on a chromosome; we have observed that approximately $5 \%$ of the normal population possess three copies of SMN1. It is therefore possible for a carrier to possess one chromosome with two copies and a second chromosome with zero copies. The finding of two SMN1 genes on a single chromosome has serious genetic counseling implications, because a carrier with two SMN1 genes on one chromosome and a SMN1 deletion on the other chromosome will have the same dosage result as a noncarrier with one SMN1 gene on each chromosome 5. Thus, the finding of normal two SMN1 copy dosage significantly reduces the risk of being a carrier; however, there is still a residual risk of being a carrier and subsequently a small recurrence risk of future affected offspring for individuals with 2 SMN1 gene copies. Risk assessment calculations using Bayesian analysis are essential for the proper genetic counseling of SMA families.

Currently, only individuals with a family history of SMA are routinely being offered carrier testing. However, more broadbased population carrier screening is currently recommended for a number of other genetic disorders with similar carrier frequencies. The prototype for heterozygote screening was testing for Tay-Sachs disease in the Ashkenazi Jewish population, where carrier testing has been offered since 1969. Carrier screening, followed by prenatal diagnosis when indicated, has resulted in a dramatic decrease in the incidence of Tay-Sachs disease in the Jewish population. It is generally accepted that the following criteria should be met for a screening program to be successful: (1) disorder is clinically severe, (2) high frequency of carriers in the screened population, (3) availability of a reliable test with a high specificity and sensitivity, (4) availability of prenatal diagnosis, and (5) access to genetic counseling. SMA fits the criteria for population-based genetic screening. Carrier screening is recommended upon the availability of educational material that can be utilized by patients and providers.
The goal of population based SMA carrier screening is to identify couples at risk for having a child with SMA. Preconception carrier screening allows carrier couples to consider the fullest range of reproductive options. The choice to have a SMA carrier test should be made by an informed decision. Educational brochures are available and provide information about SMA and the inheritance patterns. It is important for couples to understand the dosage testing. Because, SMA is the result of a common single deletion event in $95 \%$ of the cases, the carrier test is very sensitive ( $\sim 90 \%$ detection rate). However, the molecular testing does not identify all carriers and therefore false-negatives can occur. Approximately $5 \%$ of affected patients are compound heterozygotes, exhibiting a deletion and a point mutation. The dosage testing does not identify such point mutation carriers. It is well known that a false-negative result in SMA carriers occurs when the carrier has two SMN1 genes in cis on the one chromosome 5. Further, approximately $2 \%$ of affected individuals have a de novo mutation. Therefore, genetic counseling addressing specifically the possibility of false-negative results must be provided for individuals choosing carrier testing.

\section{Recommendations}

1. Because SMA is present in all populations, carrier testing should be offered to all couples regardless of race or ethnicity. Ideally, the testing should be offered before conception or early in pregnancy. The primary goal is to allow carriers to make informed reproductive choices.

2. Formal genetic counseling services must be made available to anyone requesting this testing. It is important that all individuals undergoing testing understand that a carrier is a healthy individual who is not at risk of developing the disease but has a risk of passing the gene mutation to his/her offspring. Counseling must also include a description of the disorder, including the range of severity. Educational material about SMA should be made available to all couples preferably in the preconception period. It is imperative that individuals understand the limitations of the molecular testing.

3. All identified carriers should be referred for follow-up genetic counseling for a discussion of risk to the fetus or future pregnancies. Prenatal and preimplantation diagnosis should be offered.

4. A negative screening test for one or both partners reduces but does not eliminate the possibility of an affected offspring, because the test sensitivity is $<100 \%$ ( $~ 90 \%$ detection rate). It is important that couples also recognize that the carrier testing does not provide genotype/phenotype information. Type I SMA occurs in approximately $70 \%$ of the cases, whereas the milder types II and III account for the remaining 30\% of the cases.

5. As is true for all carrier screening programs, the testing is voluntary. Informed consent and the usual caveats must be addressed including assurance of confidentiality, paternity issues, discrimination, self-esteem, and cost. 


\section{Resources}

1. Claire Altman Heine Foundation Inc., for the Prevention of Spinal Muscular Atrophy. Available at: www.prevent sma.org

2. Lefebvre S, Burglen L, Reboullet S, et al. Identification and characterization of a spinal muscular atrophy-determining gene. Cell 1995;80:155-165.

3. McAndrew PE, Parsons DW, Simard LR, et al. Identification of proximal spinal muscular atrophy carriers and patients by analysis of SMNT and SMNC gene copy number. Am J Hum Genet 1997;60:1411-1422.
4. Mailman MD, Heinz JW, Papp AC, et al. Molecular analysis of spinal muscular atrophy and modification of the phenotype by SMN2. Genet Med 2002;4:20-26.

5. Ogino S, Wilson RB. Genetic testing and risk assessment for spinal muscular atrophy (SMA). Hum Genet 2002;111:477-500.

6. Pearn J. Incidence, prevalence, and gene frequency studies of chronic childhood spinal muscular atrophy. J Med genet 1978;15:409-413.

7. Wirth B, Rudnik-Schoneborn S, Hahnen E, Rohrig D, Zerres K. Prenatal prediction in families with autosomal recessive proximal spinal muscular atrophy (5q11.2-q13.3): molecular genetics and clinical experience in 109 cases. Prenat Diagn 1995;15:407-417. 